\title{
Duty Cycle Enhancement of an Orthogonal Acceleration TOF Mass Spectrometer Using an Axially-Resonant Excitation Linear Ion Trap
}

\author{
Yuichiro Hashimoto, Hideki Hasegawa, Hiroyuki Satake, Takashi Baba, \\ and Izumi Waki \\ Central Research Laboratory, Hitachi, Ltd., Tokyo, Japan
}

\begin{abstract}
We report a new technique to enhance detection duty cycle of an orthogonal-acceleration time-of-flight mass spectrometer (oaTOF) over a broad mass range. To this end, we used an axially-resonant-excitation linear ion trap, which ejects ions axially and mass selectively into a non-mass-selective linear ion trap in front of the TOF pusher. A delay between the ejection timing of the non-mass-selective LIT and the push timing of the oaTOF was swept masssynchronously with the axially-resonant-excitation linear ion trap, so that ions are detected with duty cycle larger than $60 \%$ over a wide mass range from $\mathrm{m} / \mathrm{z} 174.1$ to 1922.0 , which is 3 to 10 times better than conventional oaTOF. (J Am Soc Mass Spectrom 2006, 17, 1669-1674) (C) 2006 American Society for Mass Spectrometry
\end{abstract}

$\mathrm{A}$ $\mathrm{n}$ orthogonal-acceleration time-of-flight mass spectrometer (hereafter oaTOF) is now a powerful tool for proteome, metabolite analyses, and other application fields where high-throughput and high mass-accuracy is required [1-3]. In addition, many hybrid oaTOF designs for tandem mass spectrometry, incorporating either a quadrupole filter or an $\mathrm{MS}^{n}$-capable ion trap, have been developed and reported [2-7]. An important performance of these standalone oaTOFs and hybrid oaTOFs is the sensitivity, because poor sensitivity would degrade the throughput of the spectrum acquisition; poor sensitivity requires long acquisition time for obtaining good $S / N$ spectrum. The sensitivity of these oaTOFs strongly depends on the "duty cycle", which is defined by the ratio of the ions accelerated by a TOF pusher to the ions introduced to the pusher. It is typically 5 to $20 \%[4,7]$ because most of the ions are lost, going through the pusher without being accelerated during the period between the acceleration pulses of the pusher. Therefore, improvement of this low duty cycle of oaTOFs is very important for enhancing the quality and the throughput of spectral acquisition.

It is well known that an ion trap whose ejection pulse is synchronized with an oaTOF can enhance the duty cycle in a limited $m / z$ range, which is often called "pulsing" technique; e.g., over $40 \%$ efficiency can be achieved between $\mathrm{m} / \mathrm{z} 300$ and 480 while compromising efficiency above $\mathrm{m} / \mathrm{z} 700$ to virtually nil [4]. In this

Published online August 22, 2006

Address reprint requests to Mr. Y. Hashimoto, Central Research Laboratory, Hitachi, Ltd., 1-280 Higashi-koigakubo, Kokubunji-shi, Tokyo 1858601, Japan. E-mail: yuichiro.hashimoto.ur@hitachi.com technique, since the ions within such a limited $\mathrm{m} / \mathrm{z}$ range travel at nearly uniform speed, one can select an optimum delay time between the trap ejection and the TOF acceleration, so that nearly all the ions within the $m / z$ range enter the TOF pusher region simultaneously and are accelerated by a single push acceleration, resulting in a high duty cycle for the limited $m / z$ range. With this method, however, the mass range of duty cycle enhancement is narrow, which is unsuitable for molecular profiling applications, such as proteome analysis.

We previously reported that an axially-resonant excitation linear ion trap, which we hereafter call AREX LIT, can mass-selectively eject the trapped ions with high efficiency of over $60 \%$ [8]. In addition, AREX LIT ejects the ions with low-energy dispersion because an axial harmonic potential is formed not by RF potential but a DC potential.

In this paper, we report that duty cycle of oaTOFs can be improved significantly by coupling an AREX LIT to an oaTOF, where high efficiency and low-energy dispersion of AREX ejection are used to mass-selectively transport ions into the oaTOF efficiently. For each $m / z$ of axial ejection from the AREX trap, the ions are then trapped in a non-mass-selective linear ion trap (LIT) in front of the oaTOF. There is a predetermined delay time between the ejection from the non-mass-selective LIT and the TOF acceleration, so that ions are detected with duty cycle of over $60 \%$. The delay time is scanned synchronously with the $m / z$ scan of AREX ejection, so that high duty cycle of TOF detection is maintained over the whole mass range of ions trapped in the AREX trap, which is 3 to 10 times better than a conventional oaTOF with a similar broad $\mathrm{m} / \mathrm{z}$ range. 


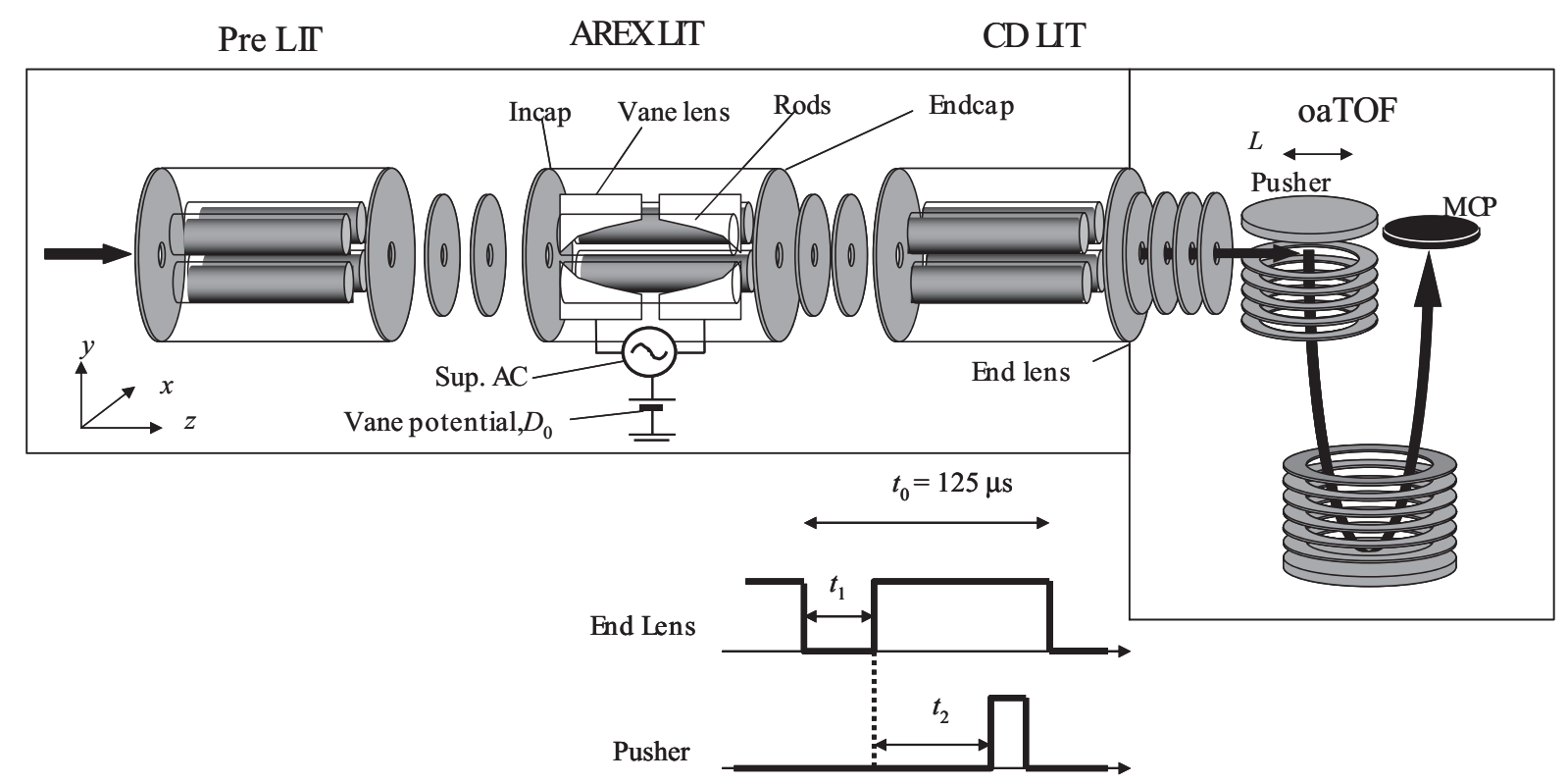

Figure 1. Schematic of an AREX LIT/oaTOF.

\section{Experimental}

The experimental setup is depicted in Figure 1. This instrument was modified from the linear ion trap/TOF mass spectrometer described previously [7]. Samples were ionized using a gas-assisted electrospray ion source at a sample flow rate of $5 \mu \mathrm{l} / \mathrm{min}$. Ions were introduced from the ESI source through high-pressure multipole rods into a first linear ion trap (hereafter pre-LIT). After accumulating ions for about $50 \mathrm{~ms}$, the ions are ejected out of the pre-LIT into an AREX LIT. In all measurement modes described below, trap/ejection operation of the pre-trap was repeated at $20 \mathrm{~Hz}$ frequency. The detail of the AREX LIT dimension was described in reference [8]. Two phases of the trapping $R F$ voltage ( $\pm 600 \mathrm{~V}$ (0-peak), $820 \mathrm{kHz})$ were applied to the rod pairs, which form a radial trapping potential. The vane potential $\left(D_{0}\right)$, which is a DC potential of the vane lenses relative to a quadrupole-rods DC offset, makes a potential that is approximately harmonic along the central axis of the quadrupole field. A supplemental AC voltage ( $< \pm 1.5 \mathrm{~V}$ (0-peak), $6-27 \mathrm{kHz}$ ) was applied between the front and rear vanes to oscillate ions resonantly in an axial direction. The oscillated ions were ejected into a non-mass-selective collisional damping LIT (hereafter CD LIT), where ions can be thermalized and collimated effectively by collisional damping [7]. The DC offset voltages of the rod electrodes for the pre-LIT, the AREX LIT, and the CD LIT were 22.0, 10.0, and $7.5 \mathrm{~V}$, respectively. The entrance lenses of each LIT were kept at DC potential between +3 and $+10 \mathrm{~V}$ relative to the DC offset voltages of the rods. To trap in the LITs, exit lenses of each LIT were kept at DC potential between of +3 and $+15 \mathrm{~V}$ relative to the DC offset voltages of the rods. To release ions from the LITs, the exit lenses were kept at DC potential between
-2 and $-10 \mathrm{~V}$ relative to the DC offset voltages of the rods. Ion signal was acquired by an analog-to-digital signal averager (FAST FLIGHT-2, ORTEC, Oakridge, $\mathrm{TN})$ to avoid counting loss by saturation of detection electronics. Acceleration frequency of the pusher was 8 $\mathrm{kHz}$; the interval of acceleration was $125 \mu \mathrm{s}$, which is independent of the LIT operation. Helium is introduced into the pre-LIT and the AREX LIT as a bath gas of 2 and 5 mTorr, respectively. Nitrogen is introduced into the CD LIT as a bath gas of 10 mTorr. Mass resolving power of the TOF was $5000 \mathrm{FWHM}$, which did not change significantly in all the measurement modes, which suggests the CD LIT could effectively focus the ion beam both spatially and energetically.

To investigate duty cycle performance, we examined the following three different modes of experimental configurations.

1. Through mode: Ions inside the pre-LIT are transferred through the AREX LIT and the CD LIT without being trapped.

2. Axially resonant ejection (AREX) mode without mass-synchronous delay (hereafter AREX mode): ions inside the pre-LIT are transferred to and trapped in the AREX LIT, from which they are mass-selectively ejected by application of axial supplemental AC. Ejected ions go through the CD LIT without being trapped.

3. AREX mode with mass-synchronous delay, [hereafter called throughput enhancement by synchronized traps (TESTs) mode]: ions inside the pre-LIT are transferred to and trapped in the AREX LIT, from which they are mass-selectively ejected by application of axial supplemental AC. Ejected ions are 


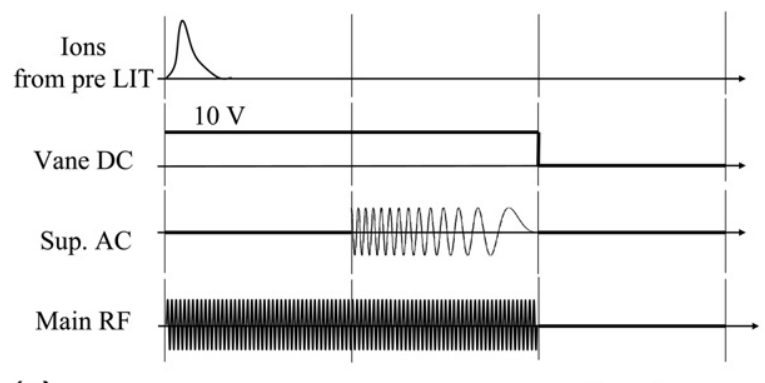

(a)

$$
\text { Accumulation Scan Emptying }
$$

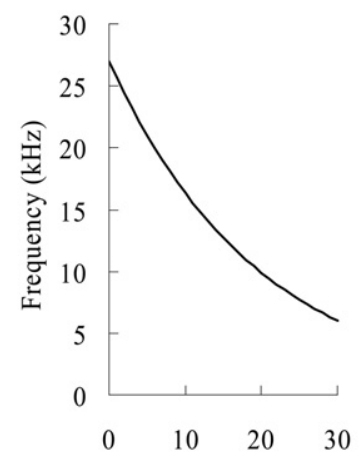

(b)

$$
\text { Time (ms) }
$$

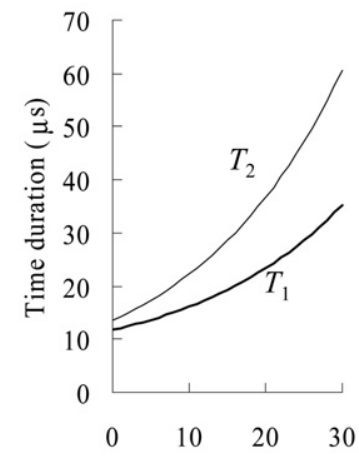

(c)

Time (ms)

Figure 2. Measurement sequence of an AREX LIT/oaTOF. (a) time sequence of AREX LIT in AREX mode and TESTs mode, (b) the frequency of the supplemental AC applied to the vane lens during scan period in AREX mode and TESTs mode, and (c) "open" duration $\left(T_{1}\right)$ of the end lens and the delay $\left(T_{2}\right)$ between the "close" time and that of the TOF acceleration during scan period in TESTs mode.

trapped in the non-mass-selective CD LIT and ejected mass-synchronously with AREX LIT.

The time sequences of the AREX mode and the TESTs mode are shown in Figure 2a. The time sequence consists of three periods for accumulation, scan, and emptying of the AREX trap. During an accumulation period of $5 \mathrm{~ms}$, the ions from the pre-LIT are accumulated inside the AREX LIT, where the vane potential of $10 \mathrm{~V}$ relative to the rod offset makes an axial trapping field, and quadrupole rods make a radial trapping field inside the trap. During the scan period of $30 \mathrm{~ms}$, a supplemental AC frequency is swept from a higher frequency to a lower one as shown in Figure 2b. A start frequency of $27 \mathrm{kHz}$ and a stop frequency of $6 \mathrm{kHz}$ correspond to $\mathrm{m} / \mathrm{z} 150$ and 3000, respectively. This achieves sequential ejection into the CD LIT from low $\mathrm{m} / \mathrm{z}$ ions to high $\mathrm{m} / \mathrm{z}$ ions. In the TESTs mode, the timing of the CD LIT's trap and ejection was controlled with the time durations as shown in Figure 2c. To enhance the ion intensity introduced into the oaTOF pusher, the time durations were controlled mass-synchronously with ejection $\mathrm{m} / \mathrm{z}$ of AREX, so that ions with a wide $\mathrm{m} / \mathrm{z}$ range between $\mathrm{m} / z 150$ and 3000 can be detected with high efficiency by the oaTOF. The optimization of duration $T_{1}$ and delay $T_{2}$ is described in the Results and Discussion section below. During an emptying period, the main AREX RF voltage was turned off, ejecting all the remaining ions out of the AREX trap.

\section{Results and Discussion}

\section{Ion Separation by the AREX LIT}

Figure 3 shows a mass spectrum, obtained in the AREX mode, of a mixture sample of a methanol solution of 200 $\mathrm{ppb}$ reserpine, $1 \mu \mathrm{M}$ glu-fibrinopeptide $\mathrm{B}$ and $10 \mathrm{ppm}$ ultramark 1621, which gives a wide $\mathrm{m} / \mathrm{z}$ range ions between $\mathrm{m} / \mathrm{z} 174.1$ and 1922.0. Since high voltage was applied to the inlet orifice for in-source CID, fragment ions such as $m / z 174.1,195.1,365.2,397.2,448.2$, and 673.4 were observed in the spectrum. The acquisition period of this spectrum was $5 \mathrm{~s}$. The maximum intensity scale of $10^{4}$ ADC counts in the figure corresponds to about 400 ion counts per second per spectral bin, where a typical reserpine ion peak at $\mathrm{m} / \mathrm{z} 609.3$ has a TOF FWHM of five bins, with one bin corresponding to 1 nano-second.

We investigated temporal characteristics of the AREX mode. Figure 4 shows temporal profile of ions detected by the oaTOF during the AREX mode, where time zero corresponds to the ejection timing of AREX, and the data were accumulated for $30 \mathrm{~s}$. Figure 4 plots the data every $500 \mu \mathrm{s}$, where spectra of four TOF pulses were summed for each data point.

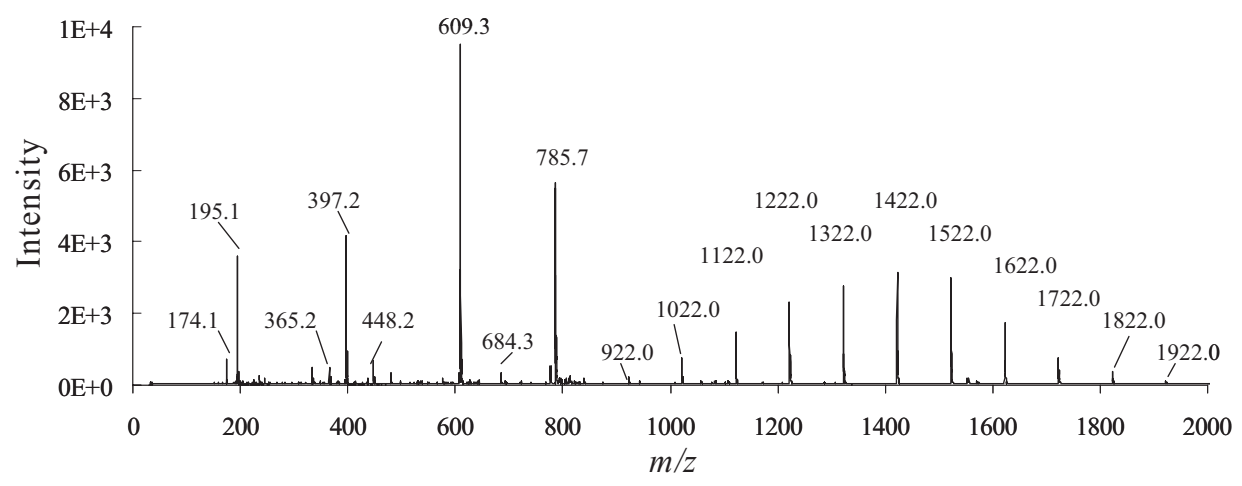

Figure 3. A mass spectrum for mixture solution of $200 \mathrm{ppb}$ reserpine, $1 \mu \mathrm{M}$ glu-fibrinopeptide $\mathrm{B}$ and 10 ppm ultramark 1621, using AREX mode. Spectrum acquisition period: $5 \mathrm{~s}$. 


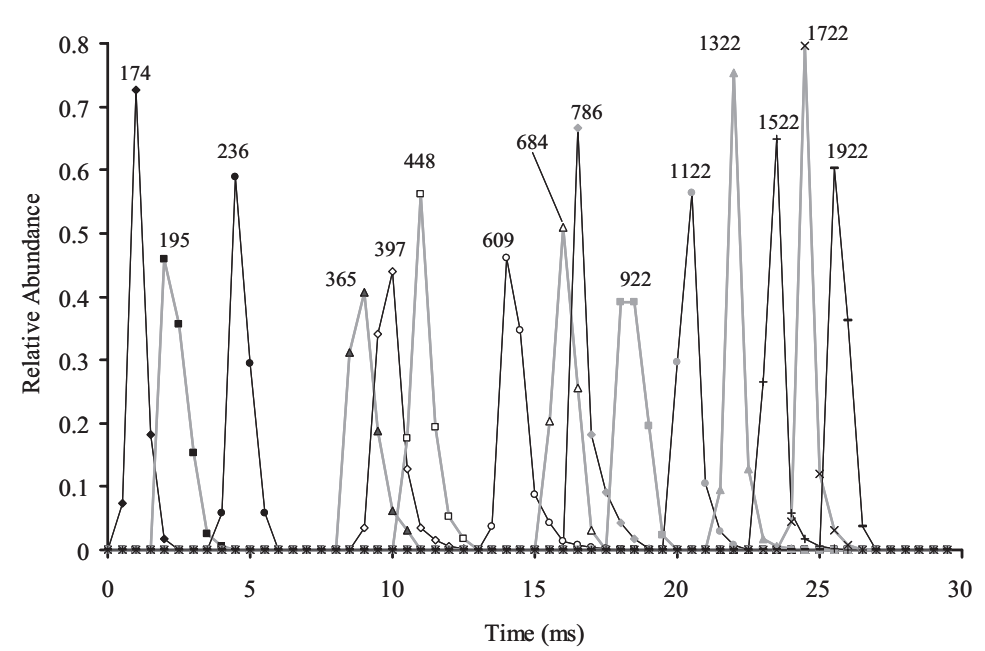

Figure 4. Mass-dependent temporal profile of ions detected by the oaTOF in the AREX mode. Start frequency $27 \mathrm{kHz}$, stop frequency $6 \mathrm{kHz}$, scan period $30 \mathrm{~ms}$, data acquisition period $30 \mathrm{~s}$. Numbers above the peaks correspond to the $\mathrm{m} / \mathrm{z}$ of the ions detected by TOF.

We adjusted the data acquisition parameters of Figure 4 , so that the time profiles are not saturated, as explained in the following. For $30 \mathrm{~ms}$ scan time from $\mathrm{m} / \mathrm{z} 174.1$ to 1922.0 , FWHM width of each species is about $1 \mathrm{~ms}$, resulting in a peak capacity of about 30 after passing through the CD LIT. Because time dispersions ejected from the AREX LIT are estimated to be between 150 and $500 \mu \mathrm{s}$ [8], we estimate that measured time widths originate mainly from collisional damping in the CD LIT. Since this AREX ejection sequence was repeated at a frequency of $20 \mathrm{~Hz}$, for every $1 \mathrm{~s}$ of data acquisition, $20 \mathrm{~ms}$ was used as an effective detectionduration of each ion species. Within this time duration of $20 \mathrm{~ms}$, there are 160 oaTOF pushes. Because the response of the ion detection system, comprising the MCP and the ADC, was linear up to about 30 ions per single acceleration pulse of the oaTOF, the detection electronics can detect ions of each species linearly up to 4800 ions per second. Thus, the maximum intensity scale in Figure 3 is less than 1/10 of the limit of the linear range. Because all signal intensities were also far below the saturation level in all the experiments, counting loss due to saturation was negligible.

\section{Duty Cycle of the TESTs Mode}

To operate the apparatus in the TESTs mode, we optimized the trapping and ejection timings $T_{1}$ and $T_{2}$ of the CD LIT using temporal profiles of Figure 4, so that maximum detection efficiency is achieved for each $m / z$ ejection of AREX; i.e., the lower the mass of the ions, the faster they move and, hence, the shorter are the optimum $T_{1}$ and $T_{2}$, as shown in Figure 2c. In this TESTs mode, the ion intensities of the mass peaks in Figure 4 were considerably enhanced. Figure 5 shows the enhancement factor of the TESTs mode compared with the AREX mode, $\frac{\mathrm{I}_{\text {TESTs }}}{\mathrm{I}_{\text {AREX }}}$, or compared to the Through mode,
$\frac{\mathrm{I}_{\text {TESTS }}}{\mathrm{I}_{\text {Through }}}$, where $I_{\text {Though }}, I_{\text {AREX }}$, and $I_{\text {TESTS }}$ are the ion intensities observed in the Through mode, the AREX mode, and the TESTs mode, respectively.

In a wide mass range between 174.1 and 1922.0, the ion intensities of TESTs mode are enhanced by factors between 3 and 10, either relative to the AREX or relative to the Through mode. In a higher $\mathrm{m} / \mathrm{z}$ range of more than $m / z$ 1000, the enhancement factors relative to the Through mode were higher than relative to the AREX mode. We suppose that this is due to the difference in the transmission efficiency between the Through mode and the AREX mode, where the low mass cut-off was $\mathrm{m} / \mathrm{z} 140$ for both modes. That is, in the AREX mode, where the trapped ions were cooled sufficiently by the buffer gas, the transmission of high $\mathrm{m} / \mathrm{z}$ ions was better than in the Through mode, where the ions were not trapped within AREX.

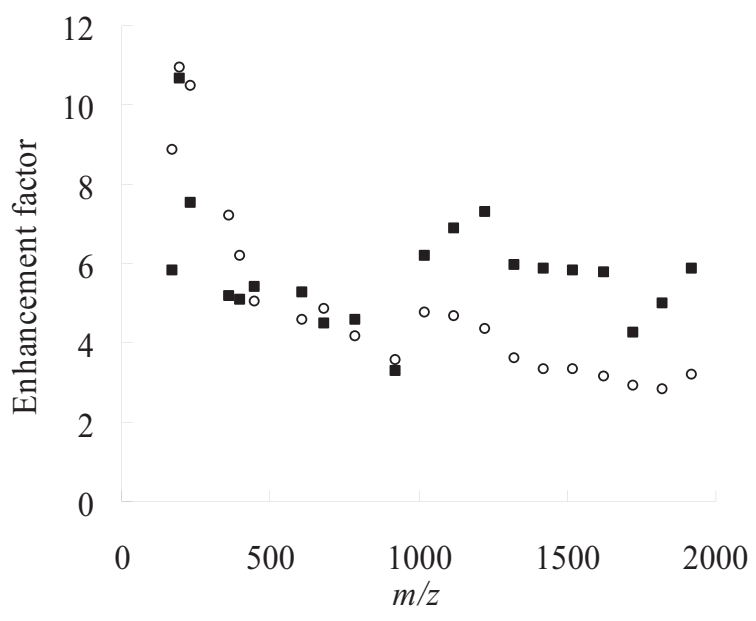

Figure 5. Enhancement factors of the TESTs mode relative to the AREX mode and the Through mode. Circles: factor relative to AREX mode. Squares: factor relative to Through mode. 


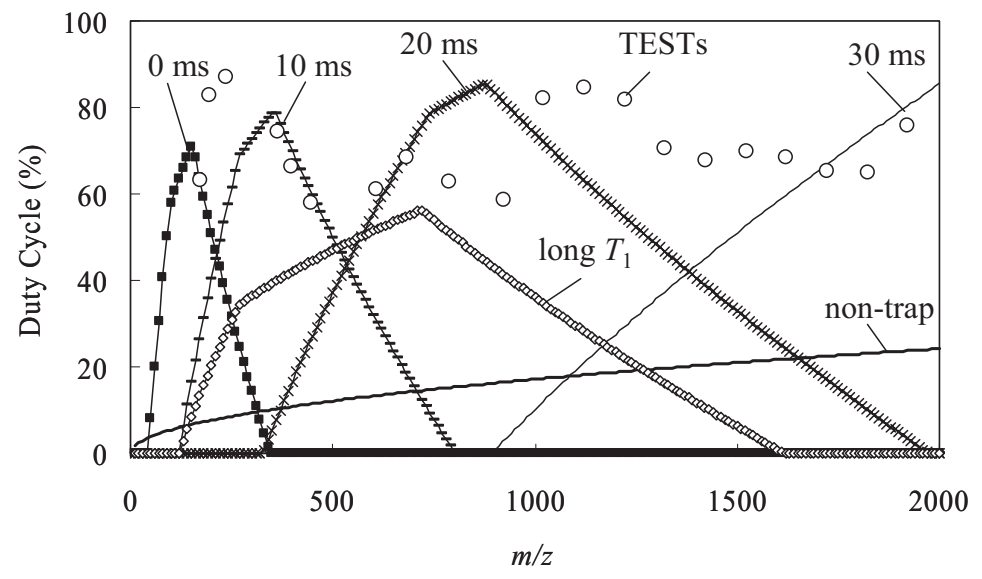

Figure 6. Comparison of duty cycles of the oaTOF for experimental TESTs data, theoretical non-trap modes, and theoretical conventional pulsing modes. Circles: experimental TESTs results obtained by eq 2. Others: theoretical simulation results where each time designation indicates the time point, or the delay from the start of AREX ejection. $0 \mathrm{~ms}: T_{1}=11.7 \mu \mathrm{s}, T_{2}=13.4 \mu \mathrm{s} .10 \mathrm{~ms}: T_{1}=16.1 \mu \mathrm{s}, T_{2}=22.2$ $\mu \mathrm{s} .20 \mathrm{~ms}: T_{1}=23.4 \mu \mathrm{s}, T_{2}=36.7 \mu \mathrm{s} .30 \mathrm{~ms}: T_{1}=35.3 \mu \mathrm{s}, T_{2}=60.6 \mu \mathrm{s}$. Long $T_{1}: T_{1}=32.1 \mu \mathrm{s}, T_{2}=$ $22.2 \mu \mathrm{s}$. Non-trap: $T_{1}=125.0 \mu \mathrm{s}, T_{2}=0.0 \mu \mathrm{s} . K=46 \mathrm{~mm} L=23 \mathrm{~mm}$, ion kinetic energy $6.0 \mathrm{eV}$.

Using the enhancement factor data shown in Figure 5 , the duty cycle of the TESTs mode, $(D C)_{T E S T s}$, was estimated by

$$
(\mathrm{DC})_{\mathrm{TESTs}}=\frac{\mathrm{I}_{\mathrm{TESTs}}}{\mathrm{I}_{\mathrm{AREX}}} \times(\mathrm{DC})_{\mathrm{AREX}}
$$

where $(D C)_{A R E X}$ is a theoretical duty cycle for the AREX mode, which is calculated under following two assumptions:

1. During the ejection duration from the CD LIT, the ion flux that is injected into the TOF acceleration region is uniform.

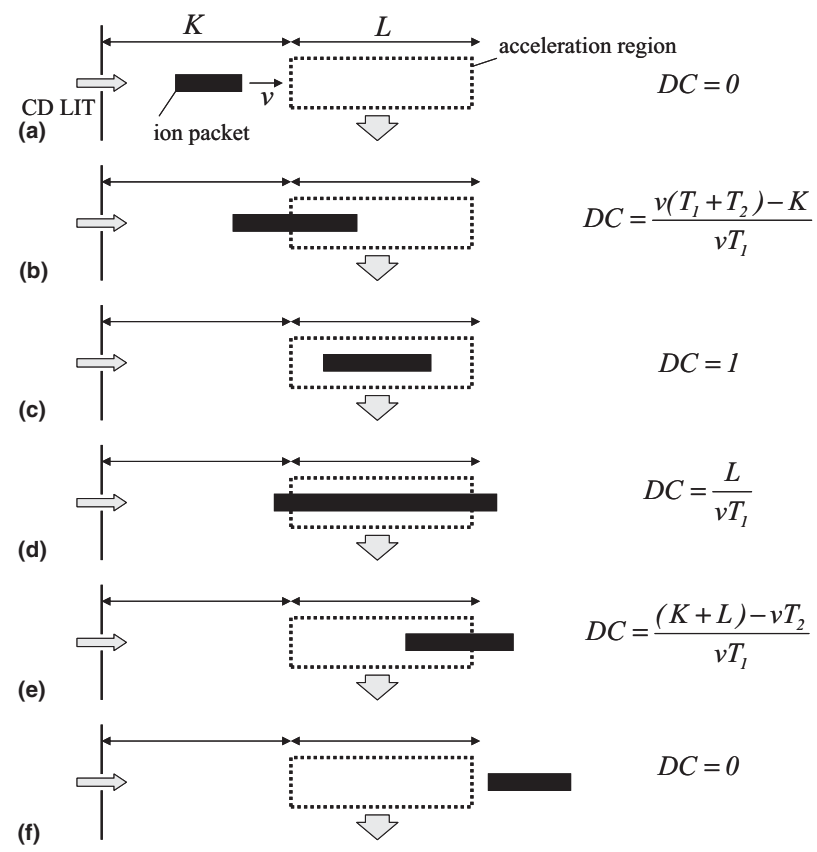

Scheme 1. Ion distribution patterns of an oaTOF.
2. Ions that are injected into the TOF acceleration region have uniform energy.

We used the enhancement factor relative to the AREX mode, instead of relative to the Through mode, because of the reduced transmission for high $\mathrm{m} / \mathrm{z}$ in the Through mode.

To calculate the theoretical duty cycles, we considered six patterns of ion distributions in the TOF acceleration region, as shown in Scheme $\mathbf{1}$, for each of which the duty cycle equation is shown as well. $K$ is the distance between the CD LIT and the entrance of the pusher, $L$ is the length of the effective acceleration region, and $v$ is the velocity of ions, which depends on the $\mathrm{m} / \mathrm{z}$ and kinetic energy. In our case, $K$ and $L$ are $46 \mathrm{~mm}$ and $23 \mathrm{~mm}$, where the ion kinetic energy was $6.0 \mathrm{eV}$.

The theoretical duty cycle of the AREX mode (or equally the Through mode), where the CD LIT does not trap ions and a continuous ion beam enters the TOF pusher, was calculated from the equation of the Scheme 1 (D), which is 7 to $24 \%$ for $m / z 174$ to 1922 and shown in Figure 6.

Figure 6 also shows the duty cycle of TESTs mode, which is obtained from the experimental enhancement factor and the theoretical duty cycle for the AREX mode together with eq 1 . High duty cycles of 60 to $90 \%$ are obtained by TESTs mode in a wide mass range between $\mathrm{m} / \mathrm{z} 174.1$ and 1922.0.

Next, we compared these experimentally-obtained TESTs duty cycle with theoretically expected duty cycle for the TESTs mode, which was calculated as follows.

During an AREX scan time of $30 \mathrm{~ms}$, four time points, which correspond to delays of $0,10,20$, and 30 ms after start of AREX ejection, are selected for theoretical calculation, for each of which optimized $T_{1}$ and $T_{2}$ are chosen as shown in Figure 2c. Then we can determine, for each $m / z$, which of the six equations in the Scheme $1(\mathrm{~A})-(\mathrm{F})$ is applicable for each time point. The 
theoretical duty cycle thus calculated with the chosen equations is shown in Figure 6. For all time points, the peak duty cycle is more than $70 \%$, but with narrow $\mathrm{m} / \mathrm{z}$ range. The $\mathrm{m} / \mathrm{z}$ range with high duty cycle increases synchronously with the AREX ejection scan. The overall envelope of the duty cycle peaks agrees reasonably well with the experimental duty cycle curve for TESTs within experimental errors.

In a conventional pulsing method [4, 9], an option exists for a compromise for increasing the $\mathrm{m} / \mathrm{z}$ range at the expense of reduced peak duty cycle by selecting a longer T1, an example of which is shown in Figure 6. In this "long T1 mode", $T_{1}$ was twice as much as the time point at $10 \mathrm{~ms}$ of Figure 6 with the same $T_{2}$. Longer duration $T_{1}$ achieves larger mass window by a factor of 2 , although the maximum duty cycle decreases by about $2 / 3$. The $m / z$ range is still narrow compared with TESTs.

\section{Conclusions}

We developed a new technique to enhance detection duty cycle of an orthogonal-acceleration time-of-flight mass spectrometer (oaTOF) over a broad mass range. With this new oaTOF, we obtained ion detection duty cycle of over $60 \%$ within a wide mass range from $\mathrm{m} / \mathrm{z} 174.1$ to 1922.0 simultaneously, which is 3 to 10 times better than conventional oaTOFs. To this end, we used an axially-resonantexcitation linear ion trap, which ejects ions axially and mass-selectively into a non-mass-selective linear ion trap in front of the TOF pusher. The delay between the ejection timing of the non-mass-selective LIT and the push timing of the oaTOF was swept mass-synchronously with the axially-resonant-excitation linear ion trap, so that ions are detected efficiently over a wide mass range. This high duty cycle should be advantageous for enhancing the quality and throughput of an orthogonal-acceleration TOF mass spectrometer in a broad range of molecular profiling applications.

\section{Acknowledgments}

The authors acknowledge the electrical supply system for this instrument provided by Mr. H. Toyama and Mr. T. Iwata of our laboratory. The authors also gratefully acknowledge the financial support provided by Hitachi High-Technologies Corporation.

\section{References}

1. Zhang, N.; Fountain, S. T.; Bi, H.; Rossi, D. T. Quantification and Rapid Metabolite Identification in Drug Discovery Using API Time-of-Flight LC/MS. Anal. Chem. 2000, 72, 800-806.

2. Morris, H. R.; Paxton, T.; Dell, A.; Langhorne, J.; Berg, M.; Bordoli, R. S.; Hoyes, J.; Bateman, R. H. High Sensitivity Collisionally-Activated Decomposition Tandem Mass Spectrometry on a Novel Quadrupole/ Orthogonal-Acceleration Time-of-Flight Mass Spectrometer. Rapid Commun. Mass Spectrom. 1996, 10, 889-896.

3. Shevchenko, A. A.; Chernushevich, I. V.; Thomson, B. A.; Ens, W.; Standing, K. G.; Wilm, M.; Mann, M. Rapid de Novo Peptide Sequencing by a Combination of Nanoelectrospray, Isotopic Labeling, and a Qquadrupole/Time-of-Flight Mass Spectrometer. Rapid Commun. Mass Spectrom. 1997, 11, 1015-1024

4. Chernushevich, I. V. Duty Cycle Improvement for a Quadrupole-Timeof-Flight Mass Spectrometer and Its Use for Precursor Ion Scans. Eur. J. Mass Spectrom. 2000, 6(6), 471-479.

5. Campbell, J. M.; Collings, B. A.; Douglas, D. J. A New Linear Ion Trap Time-of-Flight System with Tandem Mass Spectrometry CapabilitiesRapid Commun. Mass Spectrom. 1998, 12, 1463-1474.

6. Marinach, C.; Brunot, A.; Beaugrand, C.; Bolbach, G.; Tabet, J. C. Simulation of Ion Beam and Optimization of Orthogonal Tandem Ion Trap/Reflector Time-of-Flight Mass Spectrometry. Int. J. Mass Spectrom. 2002, 213, 45-62

7. Hashimoto, Y.; Hasegawa, H.; Waki, I. Dual Linear Ion Trap/Orthogonal Acceleration Time-of-Flight Mass Spectrometer with Improved Precursor Ion SelectivityRapid Commun. Mass Spectrom. 2005, 19, 14851491.

8. Hashimoto, Y.; Hasegawa, H.; Baba, T.; Waki, I. Mass Selective Ejection by Axial Resonant Excitation from a Linear Ion Trap. J. Am. Soc. Mass Spectrom. 2006, 17, 685-690.

9. Hashimoto, Y; Waki, I.; Yoshinari, K.; Shishika, T.; Terui, Y. Orthogonal Trap Time-of-Flight Mass Spectrometer Using a Collisional Damping Chamber. Rapid Commun. Mass Spectrom. 2005, 19, 221-226. 